\title{
GERSTENHABER-BATALIN-VILKOVISKI STRUCTURES ON COISOTROPIC INTERSECTIONS
}

\author{
Vladimir BARANOVSKy AND Victor GinzBURG
}

\begin{abstract}
Let $Y, Z$ be a pair of smooth coisotropic subvarieties in a smooth algebraic Poisson variety $X$. We show that any data of first order deformation of the structure sheaf $\mathcal{O}_{X}$ to a sheaf of noncommutative algebras and of the sheaves $\mathcal{O}_{Y}$ and $\mathcal{O}_{Z}$ to sheaves of right and left modules over the deformed algebra, respectively, gives rise to a Batalin-Vilkoviski algebra structure on the Tor-sheaf $\mathscr{T}_{\text {or }} \mathcal{O}_{X}\left(\mathcal{O}_{Y}, \mathcal{O}_{Z}\right)$. The induced Gerstenhaber bracket on the Tor-sheaf turns out to be canonically defined; it is independent of the choices of deformations involved. There are similar results for Ext-sheaves as well.

Our construction is motivated by, and is closely related to, a result of BehrendFantechi [2], who studied intersections of Lagrangian submanifolds in a symplectic manifold.
\end{abstract}

\section{Introduction}

1.1. Main result. Let $\mathbb{C}$ be a field of characteristic zero. We let $\mathbb{C}_{\varepsilon}:=\mathbb{C}[\varepsilon] /\left(\varepsilon^{2}\right)$ denote the ring of dual numbers and let all unlabeled tensor products stand for $\otimes_{\mathbb{C}}$. Given an algebraic variety $X$, we write $\mathcal{O}_{X}$ for the structure sheaf, resp. $T_{X}$ for the tangent sheaf on $X$.

Fix a smooth algebraic variety $X$, over $\mathbb{C}$, and $P \in H^{0}\left(X, \Lambda^{2} T_{X}\right)$, a Poisson bivector. Thus, there is a Poisson bracket $\{-,-\}: \mathcal{O}_{X} \times \mathcal{O}_{X} \rightarrow \mathcal{O}_{X}$ given by the formula $\{f, g\}=\langle P, d f \wedge d g\rangle$.

Let $\mathcal{A}$ be a sheaf of (not necessarily commutative) $\mathbb{C}_{\varepsilon}$-algebras equipped with an algebra isomorphism $\mathcal{A} / \varepsilon \mathcal{A} \sim \mathcal{O}_{X}$ so that $\mathcal{A}$ gives a flat deformation of the structure sheaf $\mathcal{O}_{X}$. We require, in addition, that the Poisson bracket induced by the commutator in $\mathcal{A}$ be equal to the bracket $\{-,-\}$. A particular example of such a deformation is the sheaf $\mathcal{A}:=\mathbb{C}_{\varepsilon} \otimes \mathcal{O}_{X}=\mathcal{O}_{X} \oplus \varepsilon \mathcal{O}_{X}$, equipped with multiplication given by the well-known formula $f \times g \mapsto f * g=f g+\frac{\varepsilon}{2}\{f, g\}$, for any $f, g \in \mathcal{O}_{X}$.

Let $Z \subset X$ be a smooth subvariety. In this paper, we are interested in flat deformations of the sheaf $\mathcal{O}_{Z}$, viewed as an $\mathcal{O}_{X}$-module supported on $Z$, to either left or right $\mathcal{A}$-module $\mathcal{C}$ set theoretically supported on $Z$. Associated with such a deformation $\mathcal{C}$ to a left $\mathcal{A}$-module, there is a transposed deformation $\mathcal{C}^{t}$, which gives a right $\mathcal{A}$-module, see section 4.1 for the definition of $\mathcal{C}^{t}$.

Next, let $Y, Z \subset X$ be a pair of smooth subvarieties. Then, $Y \cap Z$, a scheme theoretic intersection, is a closed subscheme of $X$ with structure sheaf $\mathcal{O}_{Y \cap Z}:=$ $\mathcal{O}_{Y} \bigotimes_{\mathcal{O}_{X}} \mathcal{O}_{Z}$. More generally, we have $\operatorname{Tor}^{\mathcal{O}_{X}}\left(\mathcal{O}_{Y}, \mathcal{O}_{Z}\right)$, a coherent sheaf of supercommutative graded $\mathcal{O}_{Y \cap Z}$-algebras. Similarly one has a sheaf $\mathscr{E} x t_{\mathcal{O}_{X}}\left(\mathcal{O}_{Y}, \mathcal{O}_{Z}\right)$ that

Received by the editors July 7, 2009. 
comes equipped with the natural structure of a graded $\mathscr{T}_{0 r}{ }^{\mathcal{O}}{ }_{x}\left(\mathcal{O}_{Y}, \mathcal{O}_{Z}\right)$-module (the module structure is recalled in Section 3.2).

Recall that a graded commutative algebra $D=\bigoplus_{k>0} D_{k}$ equipped with an operator $\delta: D \cdot \rightarrow D_{\boldsymbol{-}_{-1}}$ is called a Batalin-Vilkovisky (BV) algebra if $\delta$ is a differential operator of order $\leq 2$ (with respect to multiplication in $D$ ) and one has $\delta^{2}=0$. In this case, the formula (for $x, y$ homogeneous):

$$
[x, y]:=\delta(x \cdot y)-\delta(x) \cdot y-(-1)^{\operatorname{deg} x} x \cdot \delta(y),
$$

provides $D$. with a structure of Gerstenhaber algebra (i.e., odd Poisson algebra). See e.g. [2] for more details on these definitions.

Similarly, given a graded $D$-module $M=\bigoplus_{k>0} M_{k}$, a BV-module structure on $M$ is the data of a linear operator $\delta^{\prime}: M \bullet \rightarrow M_{\bullet-1}$ such that $\left(\delta^{\prime}\right)^{2}=0$ and such that $\delta^{\prime}$ has order $\leq 2$ in the sense that for any homogeneous $x, y \in D$ and $m \in M$, the following equation holds

$$
\begin{aligned}
& \delta(x y) m-(-1)^{\operatorname{deg} x} x \delta(y) m-\delta(x) y m=\delta^{\prime}(x y m)- \\
& \quad-(-1)^{\operatorname{deg} x} x \delta^{\prime}(y m)-(-1)^{\operatorname{deg} x \operatorname{deg} y+\operatorname{deg} y} y \delta^{\prime}(x m)+(-1)^{\operatorname{deg} x+\operatorname{deg} y} x y \delta^{\prime}(m) .
\end{aligned}
$$

In such a case, an analogue of formula (1.1.1) (for $\delta^{\prime}$ instead of $\delta$ ) gives a pairing $\{-,-\}: D \otimes M \rightarrow M$ that makes $M$ a Gerstenhaber module over $D$.

The main result of this paper reads

Theorem 1.1.2. Let $X$ be a smooth Poisson variety with Poisson bivector $P$, and let $\mathcal{A}$ be a flat $\mathbb{C}_{\varepsilon}$-deformation of $\mathcal{O}_{X}$ such that the commutator in $\mathcal{A}$ induces the Poisson bracket given by $P$. Let $Y, Z$ be a pair of smooth coisotropic subvarieties in $X$. Then, we have

(i) Associated with the data of a flat $\mathbb{C}_{\varepsilon}$-deformation of the sheaf $\mathcal{O}_{Y}$ to a right $\mathcal{A}$ module $\mathcal{B}$ and of the sheaf $\mathcal{O}_{Z}$ to a left $\mathcal{A}$-module $\mathcal{C}$, there is a second order differential operator $\delta: \mathscr{T}_{\text {or }}{ }^{\mathcal{O}_{X}}\left(\mathcal{O}_{Y}, \mathcal{O}_{Z}\right) \rightarrow \mathscr{T} r_{-}^{\mathcal{O}_{X}}\left(\mathcal{O}_{Y}, \mathcal{O}_{Z}\right)$ that squares to zero (i.e. makes $\mathscr{T} \boldsymbol{O}^{\mathcal{O}_{X}}\left(\mathcal{O}_{Y}, \mathcal{O}_{Z}\right)$ a $B V$ algebra) provided the first order deformations locally admit extensions to second order deformations.

(ii) The induced bracket (1.1.1) on $\mathscr{T} \boldsymbol{O}^{\mathcal{O}_{X}}\left(\mathcal{O}_{Y}, \mathcal{O}_{Z}\right)$ is independent of the choice of deformations $\mathcal{B}$ and $\mathcal{C}$.

(iii) Similarly, given an additional flat $\mathbb{C}_{\varepsilon}$-deformation of $\mathcal{O}_{Z}$ to a right $\mathcal{A}$-module $\mathcal{C}^{\prime}$, there is an associated second order differential operator $\delta^{\prime}: \mathscr{E}^{\circ} t_{\mathcal{O}_{X}}\left(\mathcal{O}_{Y}, \mathcal{O}_{Z}\right) \rightarrow$ $\mathscr{E} x t_{\mathcal{O}_{X}}^{-1}\left(\mathcal{O}_{Y}, \mathcal{O}_{Z}\right)$, such that $\left(\delta^{\prime}\right)^{2}=0$ if the first order deformations locally admit extensions to second order deformations. If $\mathcal{C}^{\prime}=\mathcal{C}^{t}$ the corresponding operator $\delta^{\prime}$ provides the sheaf $\mathscr{E} x t_{\mathcal{O}_{X}}\left(\mathcal{O}_{Y}, \mathcal{O}_{Z}\right)$ with a structure of $B V$-module over the $B V$ algebra $\mathscr{T}{ }^{\mathcal{O}^{X}}\left(\mathcal{O}_{Y}, \mathcal{O}_{Z}\right)$. Moreover, the resulting pairing

$$
\{-,-\}: \mathscr{T}_{0 r}^{\mathcal{O}_{X}}\left(\mathcal{O}_{Y}, \mathcal{O}_{Z}\right) \times \mathscr{E}_{x} t_{\mathcal{O}_{X}}\left(\mathcal{O}_{Y}, \mathcal{O}_{Z}\right) \rightarrow \mathscr{E} x t_{\mathcal{O}_{X}}^{\cdot}\left(\mathcal{O}_{Y}, \mathcal{O}_{Z}\right)
$$

is independent of the choice of deformations $\mathcal{B}$ and $\mathcal{C}$.

Since flat deformations exist locally, Theorem 1.1.2 yields the following corollary, which is the second important result of the paper.

Corollary 1.1.3. Let $Y, Z \subset X$ any pair of smooth coisotropic submanifolds of an arbitrary smooth Poisson algebraic variety $X$. Then, on $\mathscr{T o r}^{\mathcal{O}_{X}}\left(\mathcal{O}_{Y}, \mathcal{O}_{Z}\right)$ there is a canonical structure of Gerstenhaber algebra. 
Furthermore, the group $\mathscr{E} x t_{\mathcal{O}_{X}}\left(\mathcal{O}_{Y}, \mathcal{O}_{Z}\right)$ has a canonical structure of Gerstenhaber module over the Gerstenhaber algebra $\mathscr{T}{ }^{\mathcal{O}_{X}}\left(\mathcal{O}_{Y}, \mathcal{O}_{Z}\right)$.

Several examples of such BV and Gerstenhaber structures are discussed in $\S 5$ below.

Our results above were, to a great extent, inspired by the work of K. Behrend and B. Fantechi [2]. Behrend and Fantechi consider a pair $Y, Z$, of Lagrangian submanifolds in a holomorphic symplectic manifold $X$. They show that one can equip the graded algebra $\mathscr{T}_{\text {or }} \mathcal{O}_{x}\left(\mathcal{O}_{Y}, \mathcal{O}_{Z}\right)$ with a Gerstenhaber bracket, resp. the graded sheaf $\mathscr{E}_{x t_{\mathcal{O}_{X}}}\left(\mathcal{O}_{Y}, \mathcal{O}_{Z}\right)$ with a BV type differential. The approach in [2] is based on reducing the case of a general Lagrangian intersection to the special case where $X=T^{*} Y$ and $Z \subset T^{*} Y$ is the graph of a holomorphic function on $Y$ (and $Y$ is identified with the zero section of $T^{*} Y$ ).

Thus, the arguments in [2] rely in a crucial way on a version of Darboux theorem saying that any holomorphic symplectic manifold is locally isomorphic to a cotangent bundle. Such a result holds for holomorphic symplectic manifolds (equipped with the usual Hausdorff topology) but it is totally false in the algebraic setting. Indeed, an algebraic symplectic 2 -form need not be locally exact, even in ètale topology. The corresponding argument, kindly communicated to us by A. Beilinson, will be given in section 5.3.

1.2. Construction of the $\mathrm{BV}$ differential. Let $\mathcal{A}$ be any flat $\mathbb{C}_{\varepsilon}$-deformation of the sheaf $\mathcal{O}_{X}$ to a sheaf of associative $\mathbb{C}_{\varepsilon}$-algebras equipped with an algebra isomorphism $\mathcal{A} / \varepsilon \mathcal{A} \simeq \mathcal{O}_{X}$. Similarly, let $\mathcal{B}$ be a flat deformation of $\mathcal{O}_{Y}$ to a right $\mathcal{A}$-module and $\mathcal{C}$ a flat deformation of $\mathcal{O}_{Z}$ to a left $\mathcal{A}$-module. The flatness assumptions imply that multiplication by $\varepsilon$ induces an isomorphism $\mathcal{O}_{Y}=\mathcal{B} / \varepsilon \mathcal{B} \stackrel{\sim}{\rightarrow} \varepsilon$, and similar isomorphism $\mathcal{O}_{Z}=\mathcal{C} / \varepsilon \mathcal{C} \stackrel{\sim}{\rightarrow} \varepsilon \mathcal{C}$.

The short exact sequence $0 \rightarrow \varepsilon \mathcal{C} \rightarrow \mathcal{C} \rightarrow \mathcal{C} / \varepsilon \mathcal{C} \rightarrow 0$ induces a long exact sequence

$$
\ldots \rightarrow \mathscr{T}_{i+1}^{\mathcal{A}}(\mathcal{B}, \mathcal{C} / \varepsilon \mathcal{C}) \rightarrow \mathscr{T}_{i}^{\mathcal{A}}(\mathcal{B}, \varepsilon \mathcal{C}) \rightarrow \mathscr{T}_{i}^{\mathcal{A}}(\mathcal{B}, \mathcal{C}) \rightarrow \operatorname{Tor}_{i}^{\mathcal{A}}(\mathcal{B}, \mathcal{C} / \varepsilon \mathcal{C}) \rightarrow \ldots
$$

Locally, we can choose a projective resolution $P^{\bullet}$ of $\mathcal{B}$ with $\mathcal{A}$-modules, such that $P^{\bullet} / \varepsilon P^{\bullet}$ is a resolution of $\mathcal{O}_{Y}$ with projective $\mathcal{O}_{X}$-modules. Further, we have an isomorphism of functors $(\cdot) \otimes_{\mathcal{A}} \varepsilon \mathcal{C} \simeq(\cdot) \otimes_{\mathcal{A}} \mathcal{O}_{X} \otimes_{\mathcal{O}_{X}} \mathcal{O}_{Z}$ and similarly for $\mathcal{C} / \varepsilon \mathcal{C}$. We deduce canonical isomorphisms

$$
\operatorname{Tor}_{i}^{\mathcal{A}}(\mathcal{B}, \varepsilon \mathcal{C}) \simeq \mathscr{T}^{o \mathcal{O}_{X}}\left(\mathcal{O}_{Y}, \mathcal{O}_{Z}\right) \simeq \mathscr{T} r_{i}^{\mathcal{A}}(\mathcal{B}, \mathcal{C} / \varepsilon \mathcal{C})
$$

Using these isomorphisms, the connecting morphism in the long exact sequence above yields a map

$$
\delta: \mathscr{T}_{i+1}^{\mathcal{O}_{X}}\left(\mathcal{O}_{Y}, \mathcal{O}_{Z}\right) \rightarrow \mathscr{T}_{i}^{\mathcal{O}_{X}}\left(\mathcal{O}_{Y}, \mathcal{O}_{Z}\right)
$$

Similarly, suppose that we have a deformation $\mathcal{C}^{\prime}$ of $\mathcal{O}_{Z}$ to a right $\mathcal{A}$-module. Then there is a long exact sequence

$$
\ldots \rightarrow \mathscr{E} x t_{\mathcal{A}}^{i-1}\left(\mathcal{B}, \mathcal{C}^{\prime} / \varepsilon \mathcal{C}^{\prime}\right) \rightarrow \mathscr{E} x t_{\mathcal{A}}^{i}\left(\mathcal{B}, \varepsilon \mathcal{C}^{\prime}\right) \rightarrow \mathscr{E} x t_{\mathcal{A}}^{i}\left(\mathcal{B}, \mathcal{C}^{\prime}\right) \rightarrow \mathscr{E} x t_{\mathcal{A}}^{i}\left(\mathcal{B}, \mathcal{C}^{\prime} / \varepsilon \mathcal{C}^{\prime}\right) \rightarrow \ldots
$$

In particular, one has a morphism

$$
\delta^{\prime}: \mathscr{E} x t_{\mathcal{A}}^{i}\left(\mathcal{B}, \varepsilon \mathcal{C}^{\prime}\right) \simeq \mathscr{E} x t_{\mathcal{O}_{X}}^{i}\left(\mathcal{O}_{Y}, \mathcal{O}_{Z}\right) \longrightarrow \mathscr{E} x t_{\mathcal{O}_{X}}^{i+1}\left(\mathcal{O}_{Y}, \mathcal{O}_{Z}\right) \simeq \mathscr{E} x t_{\mathcal{A}}^{i+1}\left(\mathcal{B}, \mathcal{C}^{\prime} / \varepsilon \mathcal{C}^{\prime}\right)
$$

When both $\mathcal{C}$ and $\mathcal{C}^{\prime}$ are given we will assume that $\mathcal{C}^{\prime}=\mathcal{C}^{t}$ or $\mathcal{C}^{\prime}$ is transposed to $\mathcal{C}$. 
Notation. Given a vector bundle (a locally free sheaf) $E$, we write $E^{\vee}$ for the dual vector bundle. Let $\Omega_{X}$, resp. $T_{X}=\Omega_{X}^{\vee}$, denote the cotangent, resp. tangent, sheaf on a manifold $X$. Let $N_{X / Y}$ denote the normal sheaf for a submanifold $Y \subset X$.

We often abuse the notation and write $\operatorname{Tor}_{\bullet}^{X}(-,-)$ for $\operatorname{Tor}_{\bullet}^{\mathcal{O}^{X}}(-,-)$, and semilarly for Ext's.

1.3. A conjecture by physicists. Recall first that, for any (triangulated) category $\mathscr{C}$, one can define its Hochschild cohomology groups $H^{\bullet}(\mathscr{C})$.

According to A. Kapustin and L. Rozansky one has the following

Conjecture 1.3.1. To each pair $Y, Z \subset X$, of smooth Lagrangian submanifolds of a smooth algebraic symplectic variety $X$, one can associate a triangulated category $\mathscr{C} a t_{X}(Y, Z)$, cf. [7], such that the Hochschild cohomology of the category $\mathscr{C} a t_{X}(Y, Z)$ is given by

$$
H H^{\bullet}\left(\mathscr{C} a t_{X}(Y, Z)\right) \cong \mathscr{T}_{o r}{ }^{\mathcal{O}_{X}}\left(\mathcal{O}_{Y}, \mathcal{O}_{Z}\right)
$$

Moreover, the standard Gerstenhaber bracket on Hochschild cohomology goes, under the isomorphism above, to the canonical Gerstenhaber bracket on $\mathscr{T}_{\text {or }}^{\mathcal{O}^{X}}{ }^{x}\left(\mathcal{O}_{Y}, \mathcal{O}_{Z}\right)$ provided by Corollary 1.1.3.

If $X=T^{\vee} Y$ is the cotangent bundle of $Y$ and $Z=Y$ is the zero section, then one should have $\mathscr{C} a t_{X}(Y, Z)=D^{b}(\operatorname{Coh} Y)$. In this case, we have

$$
H H^{\bullet}\left(D^{b}(\operatorname{Coh} Y)\right)=H^{\bullet}\left(Y, \Lambda^{\bullet} T_{Y}\right)=\operatorname{Tor}_{\bullet}^{T^{\vee} Y}\left(\mathcal{O}_{Y}, \mathcal{O}_{Y}\right)
$$

so that the Gerstenhaber bracket is induced by the Schouten bracket on $\Lambda^{\bullet} T_{Y}$.

More generally, let $X=T^{\vee} Y$ and $Y$ be the zero section as above, and let $Z=$ $\operatorname{Graph}(d f)$ where $f \in \mathbb{C}[Y]$. Then $\mathscr{C} a t_{X}(Y, Z)$ should be the category of matrix factorizations $\left(F \underset{d^{\prime}}{\stackrel{d}{\leftrightarrows}} F^{\prime}, d \circ d^{\prime}=f \cdot \operatorname{Id}=d^{\prime} \circ d\right)$, associated with the function $f$, cf. [11].

Remark 1.3.2. Observe that the sheaves $\mathscr{T}{ }^{\mathcal{O}_{X}}\left(\mathcal{O}_{Y}, \mathcal{O}_{Z}\right)$ are related to the hyper-Tor groups $\operatorname{Tor}_{\bullet}^{X}\left(\mathcal{O}_{Y}, \mathcal{O}_{Z}\right)$ via the local-to-global spectral sequence

$$
H^{\bullet}\left(X, \mathscr{T} r_{\bullet}^{\mathcal{O}_{X}}\left(\mathcal{O}_{Y}, \mathcal{O}_{Z}\right)\right) \Longrightarrow \operatorname{Tor}_{\bullet}^{X}\left(\mathcal{O}_{Y}, \mathcal{O}_{Z}\right) .
$$

At the same time, the global hyper-Tor may also be calculated by applying $R \Gamma$ to the sheaf of DG algebras $T$. described in Section 3 below. Thus, we expect that there exists a refined version of our results, in which Gerstenhaber or Batalin-Vilkovisky structures on the cohomology sheaves of $T$. , are replaced by their "strong homotopy" versions on T. itself. In fact, the lemmas of Section 3.2 point towards such a refinement. Similar remarks apply to the Ext groups (local and global), and the polydifferential version of the resolution $E^{\bullet}$ in Section 3.

\section{Existence of first and second order deformations.}

2.1. Algebraic setup. Following Gerstenhaber, a $\mathbb{C}_{\varepsilon}$-flat deformation of an associative $\mathbb{C}$-algebra $A$ is given by a $\mathbb{C}_{\varepsilon}$-bilinear associative product structure on the vector space $A_{\varepsilon}=A \oplus \varepsilon A$ defined by

$$
\left(a_{1} \oplus 0\right) *\left(a_{2} \oplus 0\right)=a_{1} a_{2} \oplus \varepsilon \cdot \alpha_{A}\left(a_{1}, a_{2}\right), \quad a_{1}, a_{2} \in A \subset A_{\varepsilon}
$$


with $\alpha_{A}: A \otimes A \rightarrow A$ a $\mathbb{C}$-linear map. The associativity of the $*$-product is equivalent to the equation

$$
\alpha_{A}\left(a_{1} a_{2}, a_{3}\right)-\alpha_{A}\left(a_{1}, a_{2} a_{3}\right)+\alpha_{A}\left(a_{1}, a_{2}\right) a_{3}-a_{1} \alpha_{A}\left(a_{2}, a_{3}\right)=0 .
$$

Fix a $\mathbb{C}_{\varepsilon}$-flat deformation of $A$ as above. Given a right $A$-module $B$, one may consider $\mathbb{C}_{\varepsilon}$-flat extensions of the $A$-module structure on $B$ to a right $A_{\varepsilon}$-module on $B_{\varepsilon}=B \oplus \varepsilon B$. Explicitly, such an $A_{\varepsilon}$-module structure on $B_{\varepsilon}$ is determined by a bilinear map $\alpha_{B}: B \otimes A \rightarrow B$. The corresponding right $A_{\varepsilon}$-action is given by the formula

$$
(b \oplus 0) *(a \oplus 0)=b a \oplus \varepsilon \cdot \alpha_{B}(b, a), \quad a \in A, b \in B .
$$

The map $\alpha_{B}: B \otimes A \rightarrow B$ must satisfy the associativity equation

$$
\alpha_{B}\left(b a_{2}, a_{3}\right)-\alpha_{B}\left(b, a_{2} a_{3}\right)+\alpha_{B}\left(b, a_{2}\right) a_{3}-b \alpha_{A}\left(a_{2}, a_{3}\right)=0 .
$$

Further, any pair of $\mathbb{C}_{\varepsilon}$-linear automorphisms of the form

$$
A_{\varepsilon} \rightarrow A_{\varepsilon}, \quad a \mapsto a \oplus \varepsilon \beta_{A}(a) \text { and } B_{\varepsilon} \rightarrow B_{\varepsilon}, \quad b \mapsto b \oplus \varepsilon \beta_{B}(b),
$$

where $\beta_{A}: A \rightarrow A$ and $\beta_{B}: B \rightarrow B$ are $\mathbb{C}$-linear maps, induces equivalent deformations corresponding to cochains

$$
\begin{gathered}
\left(a_{1}, a_{2}\right) \mapsto \alpha_{A}\left(a_{1}, a_{2}\right)+\beta_{A}\left(a_{1} a_{2}\right)-a_{1} \beta_{A}\left(a_{2}\right)-\beta_{A}\left(a_{1}\right) a_{2} \\
\left(b, a_{2}\right) \mapsto \alpha_{B}\left(b, a_{2}\right)+\beta_{B}\left(b a_{2}\right)-b \beta_{A}\left(a_{2}\right)-\beta_{B}(b) a_{2}
\end{gathered}
$$

A deformation as above extends to $\mathbb{C}[\varepsilon] /\left(\varepsilon^{3}\right)$ if and only if one has

$$
\begin{gathered}
\alpha_{A}\left(\alpha_{A}\left(a_{1}, a_{2}\right), a_{3}\right)-\alpha_{A}\left(a_{1}, \alpha_{A}\left(a_{2}, a_{3}\right)\right)=d \gamma_{A}\left(a_{1}, a_{2}, a_{3}\right) \\
\alpha_{B}\left(\alpha_{B}\left(b, a_{1}\right), a_{2}\right)-\alpha_{B}\left(b, \alpha_{A}\left(a_{1}, a_{2}\right)\right)=d \gamma_{B}\left(b, a_{1}, a_{2}\right),
\end{gathered}
$$

where $\gamma_{A}: A \otimes A \rightarrow A, \gamma_{B}: B \otimes A \rightarrow B$ are some linear maps and $d \gamma_{A}, d \gamma_{B}$ are defined similarly to the LHS of (2.1.1) and (2.1.2), respectively.

2.2. Deformation complex. The identities of the previous subsection can be reformulated as follows. The $A$-module structure on $B$ defines a homomorphism $g: A \rightarrow$ End $_{\mathbb{C}} B$ of algebras over $\mathbb{C}$, and deforming the algebra/module structure amounts to deforming $g$ to an algebra homomorphism $A_{\varepsilon} \rightarrow \operatorname{End}_{\mathbb{C}_{\varepsilon}}\left(B_{\varepsilon}\right)$. Observe that $\operatorname{End}_{\mathbb{C}_{\varepsilon}}\left(B_{\varepsilon}\right)$ is the trivial deformation of $\operatorname{End}_{\mathbb{C}} B$. Thus, adjusting the definitions of [5], [4] (i.e. removing the term responsible for the deformation of the algebra $\operatorname{End}_{\mathbb{C}} B$ ) we introduce a deformation complex $C_{A, B}^{\bullet}$ with terms

$C_{A, B}^{n}=C^{n}(A, A) \oplus C^{n-1}\left(A, \operatorname{End}_{\mathbb{C}} B\right)=\operatorname{Hom}_{\mathbb{C}}\left(A^{\otimes n}, A\right) \oplus \operatorname{Hom}_{\mathbb{C}}\left(A^{\otimes(n-1)}, \operatorname{End}_{\mathbb{C}} B\right)$, where $C^{n}(A, X)$ denotes the standard complex of Hochschild cochains of an $A$ bimodule $X$. The differential in the complex $C_{A, B}^{\cdot}$ is given by

$$
d_{A, B}\left(\alpha_{A} \oplus \alpha_{B}\right)=d_{\text {Hоси }} \alpha_{A} \oplus\left(g \alpha_{A}-d_{\text {Hоси }} \alpha_{B}\right),
$$

where $d_{\text {Hoch }}$ stands for the standard Hochschild differential, cf. loc. cit. We put $H_{A, B}^{n}:=H^{n}\left(C_{A, B}^{\cdot}\right)$.

Equations (2.1.1), (2.1.2) say that $\alpha=\alpha_{A} \oplus \alpha_{B}$ is a cocycle in $C_{A, B}^{2}$. Equations (2.1.3) and (2.1.4) say that the equivalence class of the deformation depends only on the image of $\alpha$ in $H_{A, B}^{2}$. 
To reinterpret integrability conditions recall that by loc. cit. $H_{A, B}^{\bullet-1}$ has a structure of graded Lie algebra such that $H^{\bullet-1}(A, A)$, the Hochschild cohomology with Gerstenhaber's bracket, is a quotient Lie algebra of $H_{A, B}^{\cdot-1}$. Explicitly, up to a choice of signs, for $\alpha_{A} \oplus \alpha_{B} \in C_{A, B}^{n}, \alpha_{A}^{\prime} \oplus \alpha_{B}^{\prime} \in C_{A, B}^{m}$ one has

$$
\begin{aligned}
& {\left[\alpha_{A} \oplus \alpha_{B}, \alpha_{A}^{\prime} \oplus \alpha_{B}^{\prime}\right]=\left(\alpha_{A} \circ \alpha_{A}^{\prime}-(-1)^{(n-1)(m-1)} \alpha_{A}^{\prime} \circ \alpha_{A}\right) \oplus} \\
& \oplus\left(\alpha_{B} \circ \alpha_{A}^{\prime}+\alpha_{B} \cup \alpha_{B}^{\prime}-(-1)^{(n-1)(m-1)}\left(\alpha_{B}^{\prime} \circ \alpha_{A}+\alpha_{B}^{\prime} \cup \alpha_{B}\right)\right)
\end{aligned}
$$

where

$$
\alpha_{B} \circ \alpha_{A}^{\prime}:=\sum_{s=1}^{n}(-1)^{(s-1)(m-1)} \alpha_{B}\left(1_{A}^{\otimes(s-1)} \otimes \alpha_{A}^{\prime} \otimes 1_{A}^{\otimes(n-s)}\right)
$$

and similarly for the other terms. The cup product $\alpha_{B} \cup \alpha_{A}^{\prime}: B \otimes A^{\otimes^{m+n-1}} \rightarrow B$ is the composition of $\alpha_{B} \otimes \alpha_{A}^{\prime}: B \otimes A^{\otimes^{m+n-1}} \rightarrow B \otimes A$ and the action map $B \otimes A \rightarrow B$.

Then, equations $(2.1 .5),(2.1 .6)$ say that $\frac{1}{2}[\alpha, \alpha]=d_{A, B}(\gamma)$, i.e. that $[\alpha, \alpha]$ represents the zero class in $H_{A, B}^{3}$.

Observe that $C^{\bullet-1}\left(A\right.$, End $\left._{\mathbb{C}} B\right)$ is a subcomplex of $C_{A, B}^{\bullet}$, and $C^{\bullet}(A, A)$ is a quotient complex of $C_{A, B}^{\bullet}$. The corresponding long exact sequence of cohomology reads

$$
\ldots \rightarrow \operatorname{Ext}_{A}^{n-1}(B, B) \rightarrow H_{A, B}^{n} \rightarrow H^{n}(A, A) \stackrel{g}{\rightarrow} \operatorname{Ext}_{A}^{n}(B, B) \rightarrow \ldots
$$

We see that an $n$-cocycle $\alpha_{A} \in C^{2}(A, A)$ may be lifted to a class in $H_{A, B}^{2}$ if and only if the map $g \circ \alpha_{A}: A \times A \rightarrow \operatorname{End}_{\mathbb{C}} B$, that represents the image of the class of $\alpha_{A}$ under the connecting homomorphism, gives the zero class in $\operatorname{Ext}_{A}^{2}(B, B)$.

Similarly, given another algebra homomorphism $h: A \rightarrow\left(\operatorname{End}_{\mathbb{C}} C\right)^{o p}$, one can introduce a bigger deformation complex with terms

$$
C_{A, B, C}^{n}=C^{n}(A, A) \oplus C^{n-1}\left(A, \operatorname{End}_{\mathbb{C}} B\right) \oplus C^{n-1}\left(A, \operatorname{End}_{\mathbb{C}} C\right) .
$$

The differential in the complex $C_{A, B, C}^{\bullet}$ is given by

$$
d_{A, B, C}\left(\alpha_{A} \oplus \alpha_{B} \oplus \alpha_{C}\right)=d_{\text {Hoch }} \alpha_{A} \oplus\left(g \alpha_{A}-d_{\text {Hoch }} \alpha_{B}\right) \oplus\left(h \alpha_{A}-d_{\text {Hoch }} \alpha_{C}\right) .
$$

For the corresponding cohomology groups $H_{A, B, C}^{\bullet}$ there is a long exact sequence

$$
\begin{aligned}
\ldots \rightarrow \operatorname{Ext}_{A}^{n-1}(B, B) \oplus \operatorname{Ext}_{A}^{n-1}(C, C) & \longrightarrow H_{A, B, C}^{n} \longrightarrow \\
\longrightarrow H^{n}(A, A) & \stackrel{g \oplus h}{\longrightarrow} \operatorname{Ext}_{A}^{n}(B, B) \oplus \operatorname{Ext}_{A}^{n}(C, C) \rightarrow \ldots
\end{aligned}
$$

2.3. Local deformations. Let now $X$ be a smooth affine variety and $Y \subset X$ a smooth closed subvariety. Write $A:=\mathbb{C}[X]$, resp. $B:=\mathbb{C}[Y]$, for the corresponding coordinate rings.

A bivector $P \in H^{0}\left(X, \Lambda^{2} T_{X}\right)$ with a vanishing Schouten bracket gives a Poisson structure on $A$. We will say that $Y$ is coisotropic with respect to $P$ if $P$ projects to zero in $H^{0}\left(Y, \wedge^{2} N_{X / Y}\right)$. 
Proposition 2.3.1. The map $H_{A, B}^{\bullet} \rightarrow H^{\bullet}(A, A)$, in (2.2.2), is injective. Furthermore, for any 2-cocycle $\alpha_{A} \in C^{2}(A, A)$ the following holds:

(i) $Y$ is a coisotropic subvariety in $X$ if and only if there exists $\alpha_{B}: B \otimes A \rightarrow B$ that gives a first order deformation of $B$, i.e., if and only if there exists $\alpha_{B}$ such that the pair $\left(\alpha_{A}, \alpha_{B}\right)$ gives a 2-cocycle in the complex $C_{A, B}^{\cdot}$.

(ii) Assume that $\alpha_{A}\left(a_{1}, a_{2}\right)=\frac{1}{2}\left\langle P, d a_{1} \wedge d a_{2}\right\rangle$ with $P \in H^{0}\left(X, \wedge^{2} T_{X}\right)$. Then, in (i), one may choose $\alpha_{B}: B \otimes A \rightarrow B$ to be a sum of a bidifferential operator of bidegree $(1,1)$ and a bidifferential operator of bidegree $(0,2)$.

(iii) Assume, in addition, that the bivector $P$ has a vanishing Schouten bracket with itself: $\{P, P\}=0$. Then, there exists a symmetric bilinear map $\gamma_{A}: A \otimes A \rightarrow A$ such that equation (2.1.5) holds. If, moreover, the canonical class of $Y$ is trivial, then there exist $\alpha_{B}: B \otimes A \rightarrow B$ and $\gamma_{B}: B \otimes A \rightarrow B$ such that equations (2.1.2) and (2.1.6) hold, i.e., the map $(b, a) \mapsto b a+\varepsilon \alpha_{B}(b, a)+\varepsilon^{2} \gamma_{B}(b, a)$ gives a second order deformation of $B$.

Proof. First, note that $g \alpha_{A}=d_{\text {Hoch }} \alpha_{B}$ in $C^{2}\left(A, \operatorname{End}_{\mathbb{C}} B\right)$ means that $(2.1 .2)$ holds by definition of the maps involved. This proves (i).

To prove (ii) let $I \subset A$ denote the defining ideal of the subvariety $Y$.

First, we are going to construct a map $\alpha_{B}^{0}: B \otimes I \rightarrow B$, the restriction of the cocycle $\alpha_{B}: B \otimes A \rightarrow B$, that we are looking for, to $B \otimes I$. Observe that the cocycle equation (2.1.2) implies that, the map $\alpha_{B}^{0}$ should satisfy the following two constraints:

$$
\alpha_{B}^{0}(b a, x)-\alpha_{B}^{0}(b, a x)-b \alpha_{A}(a, x)=0 ; \quad-\alpha_{B}^{0}(b, x a)+\alpha_{B}^{0}(b, x) a-b \alpha_{A}(x, a)=0,
$$

for any $b \in B, a \in A, x \in I$.

We will define $\alpha_{B}^{0}$ to be a map of the following form:

$$
\alpha_{B}^{0}(b, x)=\rho(d b, x)+b \psi(x) ;
$$

where $\rho: \Omega_{B}^{1} \otimes\left(I / I^{2}\right) \rightarrow B$ is a $B$-bilinear map and $\psi: I / I^{2} \rightarrow B$ is a first order algebraic differential operator. In terms of $\rho$ and $\psi$, the two constraints above translate into the following pair of equations, for any $a \in A, x \in I$,

$$
\psi(a x)-a \psi(x)=1_{B} \alpha_{X}(a, x) ; \quad \rho\left(d\left(\left.a\right|_{Y}\right), x\right)=2 \cdot 1_{B} \alpha_{X}(a, x) .
$$

We remark that the second equation in (2.3.2) determines $\rho$ uniquely, since every element of $B$ is an image of some $a \in A$. Observe further that, for $a, x \in I$, we have $\alpha_{A}(a, x) \in I$ since $Y$ is a coisotropic subvariety. Hence, in this case $1_{B} \alpha_{A}(a, x)=0$. We see that we may (and will) use the second equation in (2.3.2) as a definition of $\rho$; the resulting map $\rho$ is well-defined.

Observe next that the first equation in (2.3.2) is a condition on the map $\sigma_{\psi}: I / I^{2} \otimes$ $\Omega_{B}^{1} \rightarrow B$, the principal symbol of the first order differential operator $\psi$. Specifically, the equation says that $\sigma_{\psi}(a, x)=\frac{1}{2}\left\langle\left. P\right|_{Y}, d a \wedge d x\right\rangle$ for any $a \in I, x \in B$. Again, we may (and will) use the latter equation as the definition of $\sigma_{\psi}$. The resulting symbol is well-defined since $P$ sends $I \otimes I$ to zero in $B$.

Recall next that, for any $\sigma_{\psi}$, one may find a differential operator $\psi$ that has $\sigma_{\psi}$ as its principal symbol. Indeed, let $\mathscr{D} \leq 1\left(N_{X / Y}^{\vee}, \mathcal{O}_{Y}\right)$ denote the space of first order 
algebraic differential operators $N_{X / Y}^{\vee} \rightarrow \mathcal{O}_{Y}$. The variety $Y$ being smooth and affine, one has a short exact sequence, cf. [6],

$$
0 \rightarrow \operatorname{Hom}_{\mathcal{O}_{Y}}\left(N_{X / Y}^{\vee}, \mathcal{O}_{Y}\right) \rightarrow \mathscr{D}^{\leq 1}\left(N_{X / Y}^{\vee}, \mathcal{O}_{Y}\right) \rightarrow H_{o m} \mathcal{O}_{Y}\left(N_{X / Y}^{\vee} \otimes_{\mathcal{O}_{Y}} \Omega_{Y}^{1}, \mathcal{O}_{Y}\right) \rightarrow 0
$$

where the last arrow is the principal symbol map which is, therefore, surjective.

This completes the construction of the map $\alpha_{B}^{0}: B \otimes I \rightarrow B$.

It remains to extend $\alpha_{B}^{0}$ to construct a cocycle $\alpha_{B}: B \otimes A \rightarrow B$. To that end, note that since $Y$ is smooth and affine we can choose a splitting of the short exact sequence

$$
\left.0 \rightarrow N_{X / Y}^{\vee} \rightarrow \Omega_{X}^{1}\right|_{Y} \rightarrow \Omega_{Y}^{1} \rightarrow 0 .
$$

Such a splitting yields a $B$-linear map $p: \Omega_{A}^{1} \otimes_{A} B \rightarrow I / I^{2}$. Similarly, a splitting of the projection $\left.T_{X}^{*}\right|_{Y} \rightarrow T_{Y}^{*}$ yields a $B$-linear map $q: \Omega_{B}^{1} \rightarrow \Omega_{A}^{1} \otimes_{A} B$.

Using the splittings, we define

$$
\alpha_{B}(b, a)=\rho(d b, p(d a))-\frac{1}{2} P\left(q\left(\left.d a\right|_{Y}\right), q(d b)\right)+b \psi(p(d a)) .
$$

It is clear that the resulting map $\alpha_{B}$ is an extension of $\alpha_{B}^{0}$. Furthermore, an explicit calculation using identities (2.3.2) shows that the map $\alpha_{B}$ so defined satisfies the requirements of part $(\mathrm{ii})$ of the proposition.

To prove part $($ iii $)$ we need to recall an explicit version of the Hochschild-KostantRosenberg isomorphism for Hochschild cohomology.

The Hochschild complex that we are interested in is the complex with terms $C^{k}\left(A, \operatorname{End}_{\mathbb{C}} B\right)=H_{\mathbb{C}}\left(B \otimes A^{\otimes k}, B\right)$, equipped with the Hochschild differential $d_{\text {Hoch }}$. The Hochschild-Kostant-Rosenberg theorem says that, for $Y=\operatorname{Spec} B$ smooth, one has an isomorphism Alt : $H^{k}\left(C^{\bullet}\left(A, \operatorname{End}_{\mathbb{C}} B\right)\right) \stackrel{\sim}{\rightarrow} H^{0}\left(Y, \wedge^{k} N_{X / Y}\right)$. The isomorphism is constructed as follows. Given, $\gamma \in H o m_{\mathbb{C}}\left(B \otimes A^{\otimes k}, B\right)$, one obtains, by restriction to the ideal of $Y$, a polylinear map $B \otimes I^{\otimes k} \rightarrow B$. Let $\operatorname{Alt}(\gamma)$ be the antisymmetrization of this map with respect to the last $k$ arguments. One shows, that if $\gamma$ is a Hochschild cocycle, i.e. $d_{\text {Hoch }} \gamma=0$, then $\operatorname{Alt}(\gamma)\left(b, x_{1}, \ldots, x_{k}\right)=0$ whenever $x_{i} \in I^{2}$ for at least one $i=1, \ldots, k$. It follows that the map $\operatorname{Alt}(\gamma)$ descends to a map $\operatorname{Alt}(\gamma): B \otimes \Lambda_{\mathbb{C}}^{k}\left(I / I^{2}\right) \rightarrow B$. Furthermore, the equation $d_{\text {Hoch }} \gamma=0$ insures that the resulting map is $B$-polylinear, cf. [10, Proposition 1.3.12] for a similar result in the case of Hochschild homology. We conclude that the map $\operatorname{Alt}(\gamma)$ descends to a well-defined $B$-linear map $\operatorname{Alt}(\gamma): \Lambda_{B}^{k}\left(I / I^{2}\right) \rightarrow B$. Giving such a map is the same as giving a section of $\Lambda^{k} N_{X / Y}$, and we are done.

We can now resume the proof of part (iii). First of all, we note that the existence of some $\gamma_{A}$ is well-known, cf. e.g. [8]. By skew symmetry of $\alpha_{A}=\frac{1}{2} P$ is follows immediately that $\frac{1}{2}\left(\gamma_{A}(a, b)+\gamma_{A}(b, a)\right)$ solves the same equation (2.1.5). Hence, from now on, we assume that the bilinear map $\gamma_{A}$ is symmetric.

Assume now that the canonical bundle on $Y$ is trivial and choose a trivialization, that is, a nowhere vanishing top degree differential form $\omega$ on $Y$. Let $\operatorname{Lie}_{\partial}(\omega)$ denote the Lie derivative of $\omega$ with respect to a vector field $\partial$ on $Y$. The assignment $\partial \mapsto$ $\operatorname{Lie}_{\partial}(\omega) \cdot \omega^{-1}$ gives a first order differential operator $T_{Y} \rightarrow B$. We compose this differential operator with the $B$-linear map $I / I^{2} \rightarrow T_{Y}$ given by the restriction of the bivector $P$ to $Y$. This way, we obtain a first order differential operator $\psi: I / I^{2} \rightarrow B$ that satisfies the identity $\psi(a x)-a \psi(x)=1_{B} \alpha_{X}(a, x)$ (the first equation in (2.3.2)). 
We use the above operator $\psi$ to construct a cocycle $\alpha_{B}: B \otimes A \rightarrow B$ following the procedure explained in the proof of part (ii). The resulting operator $\alpha_{B}: B \otimes A \rightarrow B$ satisfies

$$
\alpha_{B}\left(\alpha_{B}\left(b, x_{1}\right), x_{2}\right)-\alpha_{B}\left(\alpha_{B}\left(b, x_{2}\right), x_{1}\right)-2 \alpha_{B}\left(b, \alpha_{A}\left(x_{1}, x_{2}\right)\right)=0, \quad x_{1}, x_{2} \in I .
$$

To complete the proof, we have to construct an operator $\gamma_{B}: B \otimes A \rightarrow B$ that satisfies the equation

$$
d_{\text {Hoch }} \gamma_{B}\left(b, a, a^{\prime}\right)=\alpha_{B}\left(\alpha_{B}(b, a), a^{\prime}\right)-\alpha_{B}\left(b, \alpha_{A}\left(a, a^{\prime}\right)\right)+b \gamma_{A}\left(a, a^{\prime}\right),
$$

where the Hochschild differential $d_{\mathrm{Hoch}}: \operatorname{Hom}_{\mathbb{C}}(B \otimes A, B) \rightarrow \operatorname{Hom}_{\mathbb{C}}(B \otimes A \otimes A, B)$ is given by the formula $d_{\mathrm{Hoch}} \gamma_{B}\left(b, a, a^{\prime}\right):=\gamma_{B}\left(b a, a^{\prime}\right)-\gamma_{B}\left(b, a a^{\prime}\right)+\gamma_{B}(b, a) a^{\prime}$.

Let $\eta\left(b, a, a^{\prime}\right)$ denote the RHS of (2.3.4). A straightforward computation shows that $\eta$ is a Hochschild cocycle, explicitly, one has

$$
\eta\left(b a, a^{\prime}, a^{\prime \prime}\right)-\eta\left(b, a a^{\prime}, a^{\prime \prime}\right)+\eta\left(b, a, a^{\prime} a^{\prime \prime}\right)+\eta\left(b, a, a^{\prime}\right) a^{\prime \prime}=0 .
$$

We claim further that $\eta$ gives the zero class in Hochschild cohomology. To see this we use the Hochschild-Kostant-Rosenberg isomorphism. Thus, we must restrict $\eta$ to $B \otimes I \otimes I$ and compute Alt $(\eta)$. But equation (2.3.3) says that the RHS of formula (2.3.4) is symmetric in the last two arguments. We conclude that $\operatorname{Alt}(\eta)=0$. Hence, $\eta$ is a Hochschild coboundary, and part (iii) follows.

Remark 2.3.5. Here we sketch another proof of part (ii): Equation (2.1.2) says that $d \alpha_{B}=\alpha_{A} \cdot I d_{B}$ holds in $C^{2}\left(A, \operatorname{End}_{\mathbb{C}}(B)\right)$. Let $D^{\bullet}\left(A, \operatorname{End}_{\mathbb{C}}(B)\right)$ be a subcomplex of the Hochschild complex formed by cochains given by multidifferential operators. The arguments from [8], pp. 16-17 can be used to show that the cohomology groups of the complex $D^{\bullet}\left(A, \operatorname{End}_{\mathbb{C}}(B)\right)$ are $\wedge^{\bullet} N_{X / Y}$. Therefore, it follows from a version of the Hochschild-Kostant-Rosenberg result that the imbedding $\jmath: D^{\bullet}\left(A, \operatorname{End}_{\mathbb{C}}(B)\right) \hookrightarrow$ $C^{\bullet}\left(A, \operatorname{End}_{\mathbb{C}}(B)\right)$ induces an isomorphism $H^{\bullet}(\jmath)$, on cohomology.

Now, the Poisson bivector on $X$ gives a class $\alpha_{A} \cdot I d_{B} \in D^{2}\left(A, \operatorname{End}_{\mathbb{C}}(B)\right)$. Since $Y$ is coisotropic, $\jmath\left(\alpha_{A} \cdot I d_{B}\right) \in C^{2}\left(A, \operatorname{End}_{\mathbb{C}}(B)\right)$ is a coboundary. By injectivity of $H^{2}(\jmath)$ the class $\alpha_{A} \cdot I d_{B} \in D^{2}\left(A, \operatorname{End}_{\mathbb{C}}(B)\right)$ is itself a coboundary, i.e. $d \alpha_{B}=\alpha_{A} \cdot I d_{B}$ for some $\alpha_{B} \in D^{2}\left(A, \operatorname{End}_{\mathbb{C}}(B)\right)$. A separate easy calculation shows that the principal symbol of $\alpha_{B}$ is a linear combination of maps $\operatorname{Sym}^{2} \Omega_{A / \mathbb{C}} \rightarrow B$ and $\Omega_{A / \mathbb{C}} \otimes \Omega_{B / \mathbb{C}} \rightarrow B$, i.e. its component $S_{y m^{2}} \Omega_{B / \mathbb{C}} \rightarrow B$ is actually zero.

Remark 2.3.6. It can be shown using the arguments of the proof above and those of Section 4 below that the existence of a (not necessarily split) deformation for $\mathcal{O}_{Y}$ with $Y$ coisotropic, is equivalent to the vanishing of a certain class in $H^{1}\left(Y, N_{X / Y}\right)$. This class is the cup product of the Atiyah class in $H^{1}\left(Y, \Omega_{Y}^{1} \otimes_{\mathcal{O}_{Y}} \operatorname{End}\left(N_{X / Y}\right)\right)$ with the image of $P$ in $H^{0}\left(Y, T_{Y} \otimes N_{X / Y}\right)$. See Theorem 7 in [1] for more details.

\section{An algebraic construction of BV operators}

3.1. Complexes computing $\operatorname{Tor}_{\bullet}^{A}(B, C)$ and $\operatorname{Ext}_{A}^{\bullet}(B, C)$. In this subsection we fix a commutative algebra $A$ and a pair of $A$-modules $B, C$. We have associated algebra homomorphisms $g: A \rightarrow \operatorname{End}_{\mathbb{C}} B$, resp. $h: A \rightarrow$ End $_{\mathbb{C}} C$. Write $T(A)$ for the tensor algebra of the vector space $A$. 
Recall that the $A$-module $B$ admits a free bar resolution $B \otimes T(A) \otimes A \rightarrow B$, cf. [12]. Therefore $\operatorname{Tor}_{\bullet}^{A}(B, C)$ and $\operatorname{Ext}_{A}(B, C)$ can be computed as the cohomology of complexes $T$. and $E^{\bullet}$, respectively, with terms

$$
T_{i}=B \otimes A^{\otimes i} \otimes C, \quad E^{i}=\operatorname{Hom}_{k}\left(B \otimes A^{\otimes i}, C\right)
$$

The corresponding differentials, $d_{T}$ and $d_{E}$, respectively, are given by

$$
\begin{aligned}
d_{T}\left(b \otimes a_{1} \otimes \ldots \otimes a_{i} \otimes c\right) & =b a_{1} \otimes \ldots \otimes a_{i} \otimes c+(-1)^{i} b \otimes a_{1} \otimes \ldots \otimes a_{i-1} \otimes a_{i} c+ \\
& +\sum_{s=1}^{i-1}(-1)^{s} b \otimes a_{1} \otimes \ldots \otimes a_{s} a_{s+1} \otimes \ldots \otimes a_{i} \otimes c \\
d_{E} \phi\left(b \otimes a_{1} \otimes \ldots \otimes a_{i+1}\right) & =-\phi\left(b a_{1} \otimes \ldots \otimes a_{i+1}\right)+(-1)^{i-1} \phi\left(b \otimes a_{1} \otimes \ldots \otimes a_{i}\right) a_{i+1} \\
& +\sum_{s=1}^{i-1}(-1)^{s-1} \phi\left(b \otimes a_{1} \otimes \ldots \otimes a_{s} a_{s+1} \otimes \ldots \otimes a_{i+1}\right)
\end{aligned}
$$

We consider deformations of the triple $(A, B, C)$. Such a deformation is determined by an element of the deformation complex $C_{A, B, C}^{2}$ given by a cocycle $\left(\alpha_{A}, \alpha_{B}, \alpha_{C}\right)$, see $\S 2.2$. Working with $T$. we always assume that $\alpha_{B}$ gives a deformation of $B$ to a right module and $\alpha_{C}$ a deformation of $C$ to a left module.

The triple $\left(\alpha_{A}, \alpha_{B}, \alpha_{C}\right)$ induces an operation $\delta_{\alpha}: T_{i} \rightarrow T_{i-1}$ given essentially by the same formula as $d_{T}$ where $b a_{1}$ is replaced by $\alpha_{B}\left(b, a_{1}\right)$, resp. $a_{s} a_{s+1}$ is replaced by $\alpha_{A}\left(a_{s}, a_{s+1}\right)$ and $a_{i} c$ by $\alpha_{C}\left(a_{i}, c\right)$. If, in addition $\alpha_{C}^{\prime}: C \otimes A \rightarrow A$ gives a deformation to a right module, then the triple $\left(\alpha_{A}, \alpha_{B}, \alpha_{C}^{\prime}\right)$ induces an operation $\delta_{\alpha}^{\prime}: E^{i} \rightarrow E^{i+1}$ given by a formula similar to $d_{E}$; this time $\phi(X) a_{i}$ is replaced by $\alpha_{C}^{\prime}\left(\phi(X), a_{i}\right)$.

The following result is proved by direct computation

Lemma 3.1.1. Let $\delta_{\alpha}$ be the operator on $T$. constructed from a triple $\left(\alpha_{A}, \alpha_{B}, \alpha_{C}\right)$.

(1) If $\left(\alpha_{A}, \alpha_{B}\right)$ is a cocycle in $C_{A, B}^{2}$ and $\left(\alpha_{A}, \alpha_{C}\right)$ is a cocycle in $C_{h}^{2}$ then, we have

$$
\delta_{\alpha} d_{T}+d_{T} \delta_{\alpha}=0
$$

(2) If $\left(\tilde{\alpha}_{A}, \tilde{\alpha}_{B}\right)-\left(\alpha_{A}, \alpha_{B}\right)=d\left(\beta_{A}, \beta_{B}\right)$ and $\left(\tilde{\alpha}_{A}, \tilde{\alpha}_{C}\right)-\left(\alpha_{A}, \alpha_{C}\right)=d\left(\beta_{A}, \beta_{C}\right)$ then, we have

$$
\delta_{\tilde{\alpha}}-\delta_{\alpha}=d_{T} \delta_{\beta}+\delta_{\beta} d_{T}
$$

where

$$
\begin{gathered}
\delta_{\beta}\left(b \otimes a_{1} \otimes \ldots \otimes a_{n} \otimes c\right)=\beta_{B}(b) \otimes a_{1} \otimes \ldots \otimes a_{n} \otimes c+ \\
+\sum_{i}(-1)^{i} b \otimes a_{1} \otimes \ldots \otimes \beta_{A}\left(a_{i}\right) \otimes \ldots \otimes a_{n} \otimes c+(-1)^{n+1} b \otimes a_{1} \otimes \ldots \otimes a_{n} \otimes \beta_{C}(c) .
\end{gathered}
$$

(3) If (2.1.5), (2.1.6) hold (with similar equation and notation assumed for $\alpha_{C}$ ), then, we have

$$
\delta_{\alpha}^{2}=d_{T} \delta_{\gamma}+\delta_{\gamma} d_{T}
$$

Similar identities hold for the map $\delta_{\alpha}^{\prime}$ constructed from $\left(\alpha_{A}, \alpha_{B}, \alpha_{C}^{\prime}\right)$, with $d_{T}$ replaced by $d_{E}$. 
We now interpret $\delta_{\alpha}$ in the context of the long exact sequence of Section 1.2. Since $B_{\varepsilon}$ is flat over $\mathbb{C}_{\varepsilon}$ we can construct a bar resolution using tensor products over $\mathbb{C}_{\varepsilon}$ :

$$
\ldots \rightarrow B_{\varepsilon} \otimes_{\mathbb{C}_{\varepsilon}} A_{\varepsilon} \otimes_{\mathbb{C}_{\varepsilon}} A_{\varepsilon} \rightarrow B_{\varepsilon} \otimes_{\mathbb{C}_{\varepsilon}} A_{\varepsilon} \rightarrow B_{\varepsilon} \rightarrow 0
$$

where the bar differential is defined using the deformed product $A_{\varepsilon} \otimes_{\mathbb{C}_{\varepsilon}} A_{\varepsilon} \rightarrow A_{\varepsilon}$ and the deformed action $B_{\varepsilon} \otimes_{\mathbb{C}_{\varepsilon}} A_{\varepsilon} \rightarrow B_{\varepsilon}$. In particular, $\operatorname{Tor}_{i}^{A_{\varepsilon}}\left(B_{\varepsilon}, C_{\varepsilon}\right)$ is the homology of the complex with the $i$-th term

$$
T_{i}^{\varepsilon}=B_{\varepsilon} \otimes \mathbb{C}_{\varepsilon} A_{\varepsilon}^{\otimes \mathbb{C}_{\varepsilon} i} \otimes_{\mathbb{C}_{\varepsilon}} C_{\varepsilon} \simeq\left[B \otimes A^{\otimes i} \otimes C\right] \oplus \varepsilon\left[B \otimes A^{\otimes i} \otimes C\right]=T_{i} \oplus \varepsilon T_{i}
$$

It is easy to see that the differential of this complex is $d_{\varepsilon}=d+\varepsilon \delta_{\alpha}$. The spectral sequence of the filtered complex (with the two step filtration) $\varepsilon T . \subset T_{\text {. }}^{\varepsilon}$ boils down to the long exact sequence

$$
\ldots \rightarrow H_{i}\left(T_{\bullet}, d\right) \rightarrow H_{i}\left(T_{\bullet}^{\varepsilon}, d_{\varepsilon}\right) \rightarrow H_{i}\left(T_{\bullet}, d\right) \rightarrow H_{i-1}\left(T_{\bullet}, d\right) \rightarrow \ldots
$$

By definition, we have $H_{i}\left(T_{\bullet}, d\right)=\operatorname{Tor}_{i}^{A}(B, C)$. The connecting differential $\delta$ : $H_{i}\left(T_{\bullet}, d\right) \rightarrow H_{i-1}\left(T_{\bullet}, d\right)$ is computed as usual: we take a representative $x \in T_{i} \subset T_{i}^{\varepsilon}$ and assume that $d x=0$, hence $d_{\varepsilon} x \in \varepsilon T_{i} \in T_{i}^{\varepsilon}$. Then, we let $\delta(x)$ be represented by the element $\frac{1}{\varepsilon} d_{\varepsilon} x$. Because of the definition of $d_{\varepsilon}$ this is precisely $\delta_{\alpha}$.

In the case of $\operatorname{Ext}_{A}(B, C)$, we assume that both $B, C$ are deformed as right modules. Again, if $\left(\alpha_{A}, \alpha_{B}, \alpha_{C}^{\prime}\right)$ satisfies the cocycle condition determining the first order deformation, the operation $\delta_{\alpha}^{\prime}$ descends to $\delta^{\prime}$ on $\operatorname{Ext}_{A}{ }^{\circ}(B, C)$ defined in Section 1.2. In fact, $\operatorname{Ext}_{A_{\varepsilon}}^{i}\left(B_{\varepsilon}, C_{\varepsilon}\right)$ may be computed as the cohomology of the complex

$$
\begin{aligned}
E_{\varepsilon}^{i}=\operatorname{Hom}_{A_{\varepsilon}}\left(B_{\varepsilon} \otimes_{\mathbb{C}_{\varepsilon}} A_{\varepsilon}^{\otimes \mathbb{C}_{\varepsilon} i} \otimes_{\mathbb{C}_{\varepsilon}} A_{\varepsilon}, C_{\varepsilon}\right)=\operatorname{Hom}_{\mathbb{C}_{\varepsilon}}\left(B_{\varepsilon} \otimes \mathbb{C}_{\varepsilon} A_{\varepsilon}^{\otimes \mathbb{C}_{\varepsilon} i}, C_{\varepsilon}\right) & =\operatorname{Hom}_{\mathbb{C}_{\varepsilon}}\left(\left[B \otimes A^{\otimes i}\right] \oplus \varepsilon\left[B \otimes A^{\otimes i}\right], C \oplus \varepsilon C\right) \\
& =\operatorname{Hom}_{k}\left(B \otimes A^{\otimes i}, C\right) \oplus \varepsilon \operatorname{Hom}_{k}\left(B \otimes A^{\otimes i}, C\right)=: E^{i} \oplus \varepsilon E^{i} .
\end{aligned}
$$

The differential $d_{\varepsilon}$ again splits into $d_{E}+\varepsilon \delta_{\alpha}^{\prime}$. Hence, the connecting differential

$$
\operatorname{Ext}_{A}^{i-1}(B, C) \rightarrow \operatorname{Ext}_{A}^{i}(B, C)
$$

is induced by $\delta_{\alpha}^{\prime}$.

Observe that for $\delta$, resp. $\delta^{\prime}$, part (1) of the Lemma 3.1 .1 implies that $\delta_{\alpha}$, resp. $\delta_{\alpha}^{\prime}$, does descend to (co)homology. Part (2) says that the operator on cohomology does not change if $\left(\alpha_{A}, \alpha_{B}, \alpha_{C}\right)$ is replaced by $\left(\alpha_{A}, \alpha_{B}, \alpha_{C}\right)+d\left(\beta_{A}, \beta_{B}, \beta_{C}\right)$. Part (3) says that integrability conditions imply $\delta^{2}=0$, resp. $\left(\delta^{\prime}\right)^{2}=0$.

3.2. Multiplicative properties of $T_{\bullet}$ and $E^{\bullet}$. We begin with a general result.

Let $(D, d)$ be a differential associative $\mathbb{Z} / 2 \mathbb{Z}$-graded algebra with an odd differential $d$ and an odd linear map $\delta: D \rightarrow D$ such that $\delta(1)=0$. We write $|x| \in \mathbb{Z} / 2 \mathbb{Z}$ for the parity of a homogeneous element $x \in D$, and introduce the notation $[d, \delta]_{+}:=d \delta+\delta d$. Define a bracket $[-,-]: D \times D \rightarrow D$ as follows

$$
[x, y]=\delta(x y)-\delta(x) y-(-1)^{|x|} x \delta(y) \quad x, y \in D .
$$

Also, for any homogeneous elements $x, y, z \in D$, put

$$
\Xi(x, y, z):=\delta(x y z)-(-1)^{|x|} x \delta(y z)-\delta(x y) z-(-1)^{|y|(|x|-1)} y \delta(x z) .
$$


A straightforward computation yields the following result

Lemma 3.2.1. The following identities hold:

$$
\begin{gathered}
d[x, y]-[d x, y]-(-1)^{|x|}[x, d y]=[d, \delta]_{+}(x y)-[d, \delta]_{+}(x) y-x[d, \delta]_{+}(y) \\
{[x, y z]-[x, y] z-(-1)^{|y||x|} y[x, z]=\Xi(x, y, z)} \\
+(-1)^{|x|+|y|} x y \delta(z)+(-1)^{|x|} x \delta(y) z+\delta(x) y z . \\
=\delta^{2}(x y z)-z \delta^{2}(x y)-x \delta^{2}(y z)-y \delta^{2}(x z)+y z \delta^{2}(x)+x z \delta^{2}(y)+z y \delta^{2}(z) \\
+\delta(\Xi(x, y, z))-\Xi(\delta(x), y, z)-(-1)^{|x|} \Xi(x, \delta(y), z)-(-1)^{|x|+|y|} \Xi(x, y, \delta(z)) .
\end{gathered}
$$

Recall next that the algebra structure on Tor-groups may be defined using the shuffle product. In more detail, according to [12], Exercise 8.6.5, Section 8.7.5 and Lemma 8.7.15 (as well as a similar statement for Ext groups), one has

Lemma 3.2.2. (i) The algebra $\operatorname{Tor}_{.}^{A}(B, C)$ is isomorphic to the homology of the $D G$ algebra $T_{.}$, see $\S 3.1$, with the shuffle product $\bullet: T_{i} \otimes T_{j} \rightarrow T_{i+j}$ given by

$$
\begin{aligned}
\left(b \otimes a_{1} \otimes \ldots \otimes a_{i} \otimes c\right) \bullet\left(b^{\prime} \otimes a_{i+1} \otimes \ldots \otimes a_{i+j} \otimes c^{\prime}\right) & = \\
& \sum_{\sigma \in S h(i, j)}(-1)^{\sigma} b b^{\prime} \otimes a_{\sigma(1)} \otimes \ldots \otimes a_{\sigma(i+j)} \otimes c c^{\prime} .
\end{aligned}
$$

(ii) The $\operatorname{Tor}_{\bullet}^{A}(B, C)$-module $\operatorname{Ext}_{A}^{\bullet}(B, C)$ is isomorphic to the cohomology of the T.-module $E^{\bullet}$ with the action $\bullet^{\prime}: T_{i} \otimes E^{j} \rightarrow E^{j-i}$ given by

$$
\begin{aligned}
&\left(b \otimes a_{1} \otimes \ldots \otimes a_{i} \otimes c\right) \bullet^{\prime} \phi\left(b^{\prime} \otimes a_{i+1} \otimes \ldots \otimes a_{j}\right)= \\
& \sum_{\sigma \in S h(i, j)}(-1)^{\sigma} \phi\left(b b^{\prime} \otimes a_{\sigma(1)} \otimes \ldots \otimes a_{\sigma(i+j)}\right) c .
\end{aligned}
$$

In order to be able to apply Lemma 3.2.1 in the situation we are interested in, we need the following result.

Lemma 3.2.3. Let $\left(T=T\right.$., $\left.d=d_{T}\right)$ be the $D G$ algebra computing the Tor groups and let $\delta=\delta_{\alpha}$, be as in section 3.1. Then, the equation $\Xi(x, y, z)=0$ holds for all $x, y, z$ if and only if, for all $b_{1}, b_{2} \in B, a \in A$, the cochain $\alpha_{B}$ satisfies

$$
\alpha_{B}\left(b_{1} b_{2}, a\right)-b_{1} \alpha_{B}\left(b_{2}, a\right)-b_{2} \alpha_{B}\left(b_{1}, a\right)+b_{1} b_{2} \alpha_{B}(1, a)=0
$$

and the cochain $\alpha_{C}$ satisfies a similar identity.

Let $\alpha_{C}^{\prime}$ be the transposed deformation defined by the formula $\alpha_{C}^{\prime}(c, a):=-\alpha_{C}(a, c)$. Then, the above conditions also insure the following identity

$$
\delta_{\alpha}(x y) m-x \delta_{\alpha}(y) m-y \delta_{\alpha}(x) m=\delta_{\alpha}^{\prime}(x y m)-x \delta_{\alpha}^{\prime}(y m)-y \delta_{\alpha}^{\prime}(x m)+x y \delta_{\alpha}^{\prime}(m) .
$$


Proof. Let $x=b_{x} \otimes x_{1} \ldots x_{l_{x}} \otimes c_{x}$ and use the similar notation for $y, z$. If we plug in the formula for $\delta$ into the definition of $\Xi(x, y, z)$ we get three kinds of terms: those which involve $\alpha_{B}, \alpha_{A}$ and $\alpha_{C}$, respectively. For instance, the terms in $\delta(x y z)$ coming from $\alpha_{A}$, will involved tensor factors of the type

$$
\begin{gathered}
\alpha_{A}\left(x_{i}, x_{i+1}\right), \alpha_{A}\left(y_{j}, y_{j+1}\right), \alpha_{A}\left(z_{s}, z_{s+1}\right), \\
\alpha_{A}\left(x_{i}, y_{j}\right), \alpha_{A}\left(y_{j}, x_{i}\right), \alpha_{A}\left(x_{i}, z_{s}\right), \alpha_{A}\left(z_{s}, x_{i}\right), \alpha_{A}\left(y_{j}, z_{s}\right), \alpha_{A}\left(z_{s}, y_{j}\right)
\end{gathered}
$$

For $x \delta(y z)$ we need to include only those terms in which $\alpha_{A}$ is applied to $y_{j}$ ans $z_{s}$ but not to $x_{i}$, and so on. Hence the terms in $\Xi(x, y, z)$ which depend on $\alpha_{A}$ cancel out by inclusion-exclusion formula. Looking at terms which involve $\alpha_{B}$ we get for $\delta(x, y, z)$ :

$$
\alpha_{B}\left(b_{x} b_{y} b_{z}, x_{1}\right), \alpha_{B}\left(b_{x} b_{y} b_{z}, y_{1}\right), \alpha_{B}\left(b_{x} b_{y} b_{z}, z_{1}\right)
$$

For $x \delta(y z)$ we get

$$
b_{x} \alpha_{B}\left(b_{y} b_{z}, y_{1}\right)+b_{x} \alpha_{B}\left(b_{y} b_{z}, z_{1}\right)
$$

and similarly for other summands in $\Xi(x, y, z)$. Extracting the terms which only contain $x_{1}$ we get

$$
\alpha_{B}\left(b_{x} b_{y} b_{z}, x_{1}\right)-b_{y} \alpha_{B}\left(b_{x} b_{z}, x_{1}\right)-b_{z} \alpha_{N}\left(b_{x} b_{y}, x_{1}\right)+b_{y} b_{z} \alpha_{B}\left(b_{x}, x_{1}\right)=0
$$

For $b_{x}=1$ this gives (3.2.4). On the other hand, if (3.2.4) holds then

$$
\begin{aligned}
& \alpha_{B}\left(b_{x} b_{y} b_{z}, x_{1}\right)=b_{y} \alpha_{B}\left(b_{x} b_{z}, x_{1}\right)+b_{x} b_{z} \alpha_{B}\left(b_{y}, x_{1}\right)-b_{x} b_{y} b_{z} \alpha_{B}\left(1, x_{1}\right) \\
& =b_{y} \alpha_{B}\left(b_{x} b_{z}, x_{1}\right)+b_{z}\left[b_{x} \alpha_{B}\left(n_{y}, x_{1}\right)-b_{x} b_{z} \alpha_{B}\left(1, x_{1}\right)\right] \\
& =b_{y} \alpha_{B}\left(b_{x} b_{z}, x_{1}\right)+b_{z}\left[\alpha_{B}\left(b_{y} b_{x}, x_{1}\right)-b_{y} \alpha_{B}\left(b_{x}, x_{1}\right)\right]
\end{aligned}
$$

as required.

The calculation for (3.2.5) is similar: for terms involving $\alpha_{B}: B \otimes A \rightarrow B$ we get precisely (3.2.4). Comparing the terms involving $\alpha_{C}: A \otimes C \rightarrow C$ and $\alpha_{C}^{\prime}: C \otimes A \rightarrow C$ we get the condition (3.2.4) for $\alpha_{C}^{\prime}$ plus the equation

$$
c_{3} \alpha_{C}\left(a, c_{1} c_{2}\right)-c_{3} c_{2} \alpha_{C}\left(a, c_{1}\right)=-\alpha_{C}^{\prime}\left(c_{1} c_{2} c_{3}, a\right)+c_{2} \alpha_{C}^{\prime}\left(c_{1} c_{3}, a\right)
$$

Since $\alpha_{C}^{\prime}$ is transposed to $\alpha_{C}$ this equation can also be reduced to (3.2.4) for $\alpha_{C}^{\prime}$.

\section{Proofs of main results}

4.1. The transposed deformation. Fix a flat $\mathbb{C}_{\varepsilon}$-algebra deformation $\mathcal{A}$, of $\mathcal{O}_{X}$. Associated with any deformation $\mathcal{C}$, of the sheaf $\mathcal{O}_{Z}$ to a left $\mathcal{A}$-module, there is a transposed deformation $\mathcal{C}^{t}$, which gives a sheaf of right $\mathcal{A}$-modules.

To explain the definition of $\mathcal{C}^{t}$, recall first that any deformation $\mathcal{C}$ admits local $\mathbb{C}$-linear splittings (in the Zariski topology) $\mathcal{C} \simeq \mathcal{O}_{Z} \oplus \varepsilon \mathcal{O}_{Z}$. So, locally, the deformed module structure can be written in the form $(a \oplus 0) *(c \oplus 0)=a c \oplus \varepsilon \alpha(a, c)$. Furthermore, by Proposition 2.3.1 the cochain $\alpha(a, c)$ can be chosen to be an algebraic differential operator in each of its arguments (which satisfies an associativity condition recalled in Section 2). Thus, $X$ has a covering by affine open subsets $U_{i}$ and on each of them there is a splitting as above. It follows that, on each double intersection $U_{i} \cap U_{j}$, the corresponding splittings differ by an automorphism

$$
c_{1} \oplus \varepsilon c_{2} \mapsto c_{1} \oplus \varepsilon\left(\psi_{i j}\left(c_{1}\right)+c_{2}\right),
$$


where $\psi_{i j}: \mathcal{O}_{Y} \rightarrow \mathcal{O}_{Y}$ is an algebraic differential operator. The gluing condition for the locally defined cochains $\alpha_{i}(a, c)$ and $\alpha_{j}(a, c)$ reads:

$$
\alpha_{i}(a, c)-\alpha_{j}(a, c)=a \psi_{i j}(c)-\psi_{i j}(a c) .
$$

Conversely, given a covering of $X$, a collection of $\alpha_{i}$ 's describing a deformation of $\left.\mathcal{O}_{Y}\right|_{U_{i}}$ to a left module over $\left.\mathcal{A}\right|_{U_{i}}$, and a set of operators $\psi_{i j}$ such that (4.1.1) holds and, moreover, such that for each triple $(i, j, k)$, one has $\psi_{i j}+\psi_{j k}=\psi_{i k}$, gives rise to a deformation of $\mathcal{O}_{Z}$ to a left $\mathcal{A}$-module $\mathcal{C}$ if the above gluing conditions are satisfied. A similar statement holds for right $\mathcal{A}$-module deformations as well.

Now, given a left $\mathcal{A}$-module deformation $\mathcal{C}$, we define $\mathcal{C}^{t}$, the transposed right $\mathcal{A}$ module deformation, by gluing locall deformations given, on each $U_{i}$, by the formula

$$
(c \oplus 0) *(a \oplus 0)=a c \oplus-\varepsilon \alpha(a, c), \quad \text { i.e. we put } \alpha_{i}^{t}(c, a):=-\alpha_{i}(a, c) .
$$

Here, the minus signs appear since the opposite algebra $\mathcal{A}^{o p}$ may be viewed as a deformation coming from the bivector $-P$. The above defined local deformations are related, on double intersections, via the operators $\psi_{i j}^{t}:=-\psi_{i j}$.

4.2. Proof of Theorem 1.1.2(i): BV differential. Given $(\mathcal{A}, \mathcal{B}, \mathcal{C})$, a triple of deformations as in the theorem, we use the construction of the map $\delta: \mathscr{T o r}^{\mathcal{O}_{X}}\left(\mathcal{O}_{Y}, \mathcal{O}_{Z}\right)$ $\rightarrow \mathscr{T o r}_{.-1}^{\mathcal{O}_{X}}\left(\mathcal{O}_{Y}, \mathcal{O}_{Z}\right)$ from section 1.2. The construction being local, the map $\delta$ restricts to a similar map on any open affine subset of $X$. Clearly, in order to verify the required properties of $\delta$, it is sufficient to verify them for an open affine covering of $X$.

This way, we reduce the proof to the case where $X=\operatorname{Spec} A$, resp. $Y=\operatorname{Spec} B$ and $Z=\operatorname{Spec} C$. For affine varieties all the deformations involved are automatically split over $\mathbb{C}$. It follows that these deformations may be written in terms of certain cocycles $\alpha_{A}, \alpha_{B}$, and $\alpha_{C}$, respectively, as in Section 2.1. Now, we are in the setting of Sections 3.2-3.3. In particular, we may use the DG algebra $\left(T_{\bullet}, d_{T}\right)$ and Lemma 3.2.2 for computing the algebra $\operatorname{Tor}_{.}^{A}(B, C)$ and we may interpret the map $\delta$ in terms of the Bar construction.

Observe next that, thanks to Lemma 3.1.1(2), replacing deformations by equivalent deformations doesn't affect the conclusion of the theorem. Therefore, we may adjust our cocycles using Proposition 2.3.1 to insure that: (1) the cocycle $\alpha_{A}$ be equal to the Poisson bracket, in particular, it can be extended to a second order deformation $\left(\alpha_{A}, \gamma_{A}\right)$; and (2) the cocycle $\alpha_{B}$, resp. $\alpha_{C}$, can also be extended to a second order deformation and, moreover, it is given by a bidifferential operator, as in Proposition 2.3.1(ii). Here, the existence of extensions to second order deformations means that there exist $\gamma_{B}$ and $\gamma_{C}$ such that for $\alpha:=\alpha_{A} \oplus \alpha_{B} \oplus \alpha_{C}$, in the deformation complex $C_{A, B, C}^{\cdot}$, see (2.2.3), we have $[\alpha, \alpha]=d_{A, B, C}\left(\gamma_{A} \oplus \gamma_{B} \oplus \gamma_{C}\right)$; in particular, the second order deformations of $B$ and $C$ given by $\left(\alpha_{B}, \gamma_{B}\right)$ and $\left(\alpha_{C}, \gamma_{C}\right)$, respectively, correspond to the same second order deformation of $A$ given by a pair $\left(\alpha_{A}, \gamma_{A}\right)$, as guaranteed by Proposition 2.3.1 (iii).

Recall that the existence of a second order extension of the deformation given by $\left(\alpha_{A}, \alpha_{B}\right)$ is equivalent to equations (2.1.5)-(2.1.6). Hence, we deduce $\left[d_{T}, \delta\right]_{+}=0$, by Lemma 3.1.1(1). Also, equation $\delta^{2}=0$ follows from Lemma 3.1.1(3). Further, the constraints from Proposition 2.3.1(ii) on the order of bidifferential operators insure that (3.2.4) holds, hence $\Xi(x, y, z)=0$ by Lemma 3.2.3. Thus, combining Lemma 
3.2.3 with Lemma 3.2.1, we deduce that the Poisson and Jacobi identities hold already in the algebra $T$. . We conclude that these identities hold in the Tor algebra as well, and the theorem follows.

The proof of the properties of the differential $\delta^{\prime}$ on the Ext-sheaves is completely similar and is left for the reader.

4.3. Proof of Theorem 1.1.2(ii)-(iii): Gerstenhaber structures. Let $Y, Z$ be smooth coisotropic submanifolds of a smooth Poisson variety $X$. We must prove the following

Theorem 4.3.1. The sheaf of graded algebras $\mathscr{T} r^{\mathcal{O}_{X}}\left(\mathcal{O}_{Y}, \mathcal{O}_{Z}\right)$ admits a canonical structure of a Gerstenhaber algebra, i.e. a graded symmetric bracket of degree (-1) which satisfies Poisson and Jacobi identities.

Similarly, the sheaf $\mathscr{E}^{x} t_{\mathcal{O}_{X}}\left(\mathcal{O}_{Y}, \mathcal{O}_{Z}\right)$ has a canonical structure of a Gerstenhaber module, i.e. a bracket $[\cdot, \cdot]^{\prime}: \mathscr{T}_{o r} \otimes \mathscr{E} x t^{j} \rightarrow \mathscr{E} x t^{j-i+1}$ such that

$$
\begin{gathered}
{[x y, m]^{\prime}=x[y, m]^{\prime}+(-1)^{\operatorname{deg} y \operatorname{deg} x} y[x, m]^{\prime} ;} \\
{[x, y m]^{\prime}=[x, y] m+(-1)^{\operatorname{deg} y \operatorname{deg} x} y[x, m]^{\prime}} \\
{[[x, y], m]^{\prime}=\left[x,[y, m]^{\prime}\right]^{\prime}+(-1)^{\operatorname{deg} y \operatorname{deg} x}\left[y,[x, m]^{\prime}\right]^{\prime}}
\end{gathered}
$$

Proof. Let $\mathcal{A}$ be the standard (split) algebra deformation of the structure sheaf $\mathcal{O}_{X}$ given by the formula $f * g=f g+\frac{\varepsilon}{2}\{f, g\}$.

On $X$, we choose an affine open covering $\left\{U_{i}\right\}$ such that each open subset $U_{i}$ has trivial canonical class. By Proposition 2.3.1(iii), on each $U_{i}$, we can find deformations $\mathcal{B}_{i}, \mathcal{C}_{i}$ which extend to second order deformations. Writing $A_{i}$, resp. $B_{i}, C_{i}$, for the corresponding algebras of global sections and applying Theorem 1.1.2, we get a BV algebra structure on Tor. $_{\bullet}^{A_{i}}\left(B_{i}, C_{i}\right)$. By Lemma 3.1.1(2), the corresponding BV differential $\delta_{i}$ on the Tor algebra is unaffected by a change of cocycles $\alpha_{A}, \alpha_{B}, \alpha_{C}$ provided neither the cohomology class of $\alpha_{A} \oplus \alpha_{B}$ nor the cohomology class of $\alpha_{A} \oplus \alpha_{C}$ is changed. In particular, we may insure that all the cocycles involved satisfy the conclusions of Proposition 2.3.1.

Next, fix a double intersection $U_{i} \cap U_{j}$. We must show that the restrictions to $U_{i} \cap U_{j}$ of the two BV differentials $\delta_{i}$ and $\delta_{j}$, arising from the triples $\left(\alpha_{A}\right)_{i},\left(\alpha_{B}\right)_{i},\left(\alpha_{C}\right)_{i}$ and $\left(\alpha_{A}\right)_{j},\left(\alpha_{B}\right)_{j},\left(\alpha_{C}\right)_{j}$ respectively, induce the same Gerstenhaber bracket on the sheaf $\left.\mathscr{T} \operatorname{Or}^{\mathcal{O}_{X}}\left(\mathcal{O}_{Y}, \mathcal{O}_{Z}\right)\right|_{U_{i} \cap U_{j}}$.

To this end, we observe that the deformation $\mathcal{A}$ being globally defined and globally split, on $U_{i} \cap U_{j}$, we have $\left(\alpha_{A}\right)_{i}=\left(\alpha_{A}\right)_{j}$. Next, consider the complex $C_{A, B}^{\cdot}$, cf. (2.2.1), associated with the algebras $A_{i j}=\mathbb{C}\left[U_{i} \cap U_{j}\right]$ and $B_{i j}=\mathbb{C}\left[Y \cap U_{i} \cap U_{j}\right]$. The injectivity claim at the beginning of Proposition 2.3.1 implies that the cocycles $\left(\alpha_{A}\right)_{i} \oplus\left(\alpha_{C}\right)_{i}$ and $\left(\alpha_{A}\right)_{j} \oplus\left(\alpha_{C}\right)_{j}$ give equal cohomology classes in $H^{2}\left(C_{\dot{A}, B}\right)$. We conclude that there exist cochains $\left(\left(\beta_{A}\right)_{i j},\left(\beta_{B}\right)_{i j}\right)$ such that, in $C_{A, B}^{2}$, we have

$$
\left(0 \oplus\left(\alpha_{C}\right)_{i}-\left(\alpha_{C}\right)_{j}\right)=d_{A, B}\left(\left(\beta_{A}\right)_{i j} \oplus\left(\beta_{B}\right)_{i j}\right) .
$$

We see that adjusting the restriction of the triple $\left(\alpha_{A}\right)_{i},\left(\alpha_{B}\right)_{i},\left(\alpha_{C}\right)_{i}$ to $U_{i} \cap U_{j}$ by a coboundary allows to achieve that $\left(\alpha_{C}\right)_{i}=\left(\alpha_{C}\right)_{j}$. 
Remark 4.3.3. At this point, the reader should be alerted that, although an equation similar to (4.3.2) holds for the difference $0 \oplus\left(\alpha_{B}\right)_{i}-\left(\alpha_{B}\right)_{j}$ as well, that equation may require a completely different choice of the cocycle $\left(\beta_{A}\right)_{i j}$. Thus, on $U_{i} \cap U_{j}$, the cocycles in the complex $C_{A, B, C}$, cf. (2.2.3), corresponding to $\left(\alpha_{A}\right)_{i} \oplus\left(\alpha_{B}\right)_{i} \oplus\left(\alpha_{C}\right)_{i}$ and $\left(\alpha_{A}\right)_{j} \oplus\left(\alpha_{B}\right)_{j} \oplus\left(\alpha_{C}\right)_{j}$ respectively, do not necessarily give equal cohomology classes, in general.

The arguments above show that we may assume, adjusting by coboundaries if necessary, that we have $\left(\alpha_{A}\right)_{i}=\left(\alpha_{A}\right)_{j}$ and $\left(\alpha_{C}\right)_{i}=\left(\alpha_{C}\right)_{j}$ (but not also $\left(\alpha_{B}\right)_{i}=\left(\alpha_{B}\right)_{j}$ at the same time). Further, let $C_{A, B}^{\cdot}$ be the deformation complex for the pair $(A, B)$. Taking the difference of the cocycles $\left(\alpha_{A}\right)_{i} \oplus\left(\alpha_{B}\right)_{i}$ and $\left(\alpha_{A}\right)_{j} \oplus\left(\alpha_{B}\right)_{j}$ yields a cocycle in $C_{A, B}$ of the form $0 \oplus\left(\alpha_{B}\right)_{i}-\left(\alpha_{B}\right)_{j}$. Since the first component here is 0 , the cocycle condition says, cf. (2.2.1), that $\left(\alpha_{B}\right)_{i}-\left(\alpha_{B}\right)_{j}: B \otimes A \rightarrow B$ is a Hochschild 1-cocycle. But any Hochschild 1-cocycle has the form $(b \otimes a) \mapsto b \cdot \xi(a)$ for a certain derivation $\xi: A_{i j} \rightarrow A_{i j}$. Thus, the difference of two BV differentials $\delta_{i}-\delta_{j}$ on $U_{i} \cap U_{j}$ is induced by the operator

$$
\tilde{\delta}\left(b \otimes a_{1} \otimes \ldots \otimes a_{i} \otimes c\right)=b \cdot \xi\left(a_{1}\right) \otimes a_{2} \otimes \ldots \otimes a_{i} \otimes c
$$

It is straightforward to verify that, since $\xi$ is a first order differential operator, any operator $\tilde{\delta}$ as above induces the zero bracket on Tor. Hence the brackets induced by $\delta_{i}$ and $\delta_{j}$ agree on $U_{i} \cap U_{j}$. This means that the Gersenhaber bracket is independent on the local choice of $\alpha_{B}$ and $\alpha_{C}$, which finishes the proof of the first part of the theorem.

We turn to the second part of the theorem concerning Gerstenhaber modules. Observe that the module bracket can be defined via

$$
[x, m]^{\prime}=\delta^{\prime}(x m)-\delta(x) m-(-1)^{\operatorname{deg} x} x \delta^{\prime}(m) .
$$

Since arbitrary $\mathbb{C}$-linear maps $B \otimes A \rightarrow B$, etc., do not localize in general, we need to work with the subcomplex of $E^{\bullet}$ given by polydifferential operators (by the last remark of Section 2 this subcomplex has the same cohomology). Observe that our deformations of $Y$ and $Z$ are indeed given locally by bidifferential operators.

Now, both versions of Poisson identity are equivalent to (3.2.5). The Jacobi identity on the module follows from $\left(\delta^{\prime}\right)^{2}=0$, once the Poisson identity is established, cf. Lemma 3.2.1(3). The remaning part of the proof is the same as for Tor.

\section{Examples.}

The proof of the previous section shows that sometimes the BV structure on Tor or Ext is well-defined globally. This is the case, for instance, whenever in the setting of the proof of Theorem 4.3.1, the cocycles $\alpha_{C}$ and $\alpha_{B}$, are defined globally.

Here is one such example.

5.1. Koszul bracket. Take $X=Y \times Y$ and view $Y \subset X$ as the diagonal. Then, one easily finds

$$
\mathscr{T o r}_{\cdot}^{\mathcal{O}_{X}}\left(\mathcal{O}_{Y}, \mathcal{O}_{Y}\right) \simeq \Omega_{Y}^{\bullet} ; \quad \mathscr{E}^{*} t_{\mathcal{O}_{X}}\left(\mathcal{O}_{Y}, \mathcal{O}_{Y}\right) \simeq \Lambda^{\bullet} \Omega_{Y}^{\vee}
$$


Proposition 5.1.1. For any Poisson bivector field $P \in H^{0}\left(Y, \wedge^{2} T_{Y}\right)$, the induced $B V$ differential $\delta$ on $\Omega_{B / k}^{\cdot}$ is given by the formula $\delta=i_{P} d_{D R}+d_{D R} i_{P}$, where the de Rham differential $d_{D R}$ has degree +1 , and where $i_{P}$ is a degree $(-2)$ contraction operation (with the bivector $P$ ). The $B V$ differential $\delta^{\prime}$ on $\Lambda^{\bullet} \Omega_{B / k}^{\vee}$ is given by the Schouten bracket $[P,-]$.

Proof. To simplify notation we will work in the affine case although all formulas make sense globally. Thus we consider $A=B \otimes B$ with the quotient map $m: B \otimes B \rightarrow B$ given by multiplication. Also, take $C=B$. By standard results, e.g. [12], we have a Hochschild cocycle on $A$ :

$$
\alpha_{A}\left(x \otimes x^{\prime}, y \otimes y^{\prime}\right)=\frac{1}{2} P(d x \wedge d y) \otimes x^{\prime} y^{\prime}-x y \otimes \frac{1}{2} P\left(d x^{\prime} \wedge d y^{\prime}\right)
$$

and the diagonal $Y_{\Delta} \subset X=Y \times Y$ is coisotropic with respect to the corresponding Poisson structure. We also have a right deformation of $B$ induced by the cocycle

$$
\alpha_{B}(b, x \otimes y)=-\frac{1}{2} P(d(b x) \wedge d y)+\frac{1}{2} P(d b \wedge d x) y: B \otimes A \rightarrow B
$$

For the second argument of $\operatorname{Tor}_{\bullet}^{A}(\cdot, \cdot)$ we use the transposed map $\alpha_{B}^{t}: A \otimes B \rightarrow B$. Our goal is to compute the induced BV differential on $\Omega_{B / k}^{*}$. To that end, we need explicit quasi-isomorphisms between $\Omega_{B / k}^{\cdot}$ and $T_{\bullet}$.

Observe that usually $\Omega_{B / k}^{\bullet}$ is identified with the cohomology of $C \cdot(A, A)=A^{\otimes(\bullet+1)}$ and the standard Hochschild differential. Our complex $T$. is slightly different although quasi-isomorphic to $C$. $(A, A)$ by the map:

$$
b \otimes a_{1} \otimes \ldots \otimes a_{n} \otimes b^{\prime} \mapsto\left(b^{\prime} b\right) \otimes m\left(a_{1}\right) \otimes \ldots \otimes m\left(a_{n}\right)
$$

Combining with the Hochschild-Kostant-Rosenberg isomorphism we have a pair of mutually inverse quasi-isomorphisms:

$$
b \otimes a_{1} \otimes \ldots \otimes a_{n} \otimes b^{\prime} \mapsto \frac{1}{n !} b^{\prime} b \cdot d m\left(a_{1}\right) \wedge \ldots \wedge d m\left(a_{n}\right): T . \rightarrow \Omega_{B / k}^{\cdot}
$$

respectively the map

$$
b_{0} d b_{1} \mapsto b_{0} \otimes\left(b_{1} \otimes 1\right) \otimes 1: \Omega_{B / k}^{1} \rightarrow T_{1}
$$

extended multiplicatively. For example, $b_{0} d b_{1} \wedge d b_{2} \wedge d b_{3}$ maps to the antisymmetrization of

$$
b_{0} \otimes\left(b_{1} \otimes 1\right) \otimes\left(b_{2} \otimes 1\right) \otimes\left(b_{3} \otimes 1\right) \otimes 1 \in T_{3}
$$

in the three middle terms. The assertion follows from the above definitions of $\alpha_{A}$ and $\alpha_{B}$ and a straightforward computation.

The case of $\delta^{\prime}$ is entirely similar.

We observe here that the differential $\delta$ of the above proposition was first constructed from a Poisson bivector $P$ by Koszul in [9]. Also, the differential $\delta$ (and not just the induced Gerstenhaber bracket) is canonically defined since the two arguments of Tor. $^{A}(B, B)$ are taken with their conjugate deformations. We also remark that the (co)homology of the differentials $\delta^{\prime}$ and $\delta$ in this case are called Poisson cohomology and homology, respectively. 
5.2. Self-intersection of a coisotropic submanifold. Let $X$ be an arbitrary Poisson variety, and let $Y=Z$, a coisotropic subvariety. In this case, we have $\mathscr{E} x t_{O_{X}}^{\circ}\left(\mathcal{O}_{Y}, \mathcal{O}_{Y}\right)=\Lambda^{\bullet}\left(N_{X / Y}\right)$.

The proof of Theorem 4.3.1 shows that the differential $\delta^{\prime}$ is well-defined globally: although $\alpha_{B}(b, a)$ exist in general only locally, on double intersections of an affine cover the difference between two choices of $\alpha_{B}$ is a coboundary (since $B=C$, where $B$ is the affine counterpart of $\mathcal{O}_{Y}$ and $C$ is the affine counterpart of $\mathcal{O}_{Z}$, as usual). Hence these two choices give the same $\delta^{\prime}$ on cohomology.

The subsheaf $\mathcal{K} \subset \boldsymbol{\Lambda}^{\bullet}\left(T_{X}\right)$ formed by vector fields which project to zero in $\Lambda^{\bullet}\left(N_{X / Y}\right)$, is a Lie subalgebra with respect to the Schouten bracket. Then $\mathcal{K}$ acts on $\Lambda^{\bullet}\left(N_{X / Y}\right)$ since any Lie subalgebra acts on a quotient by itself. Note that $P$ is a section of $\mathcal{K}$.

Proposition 5.2.1. The $B V$ differential on $\Lambda^{\bullet}\left(N_{X / Y}\right)$ is given by the action of the Poisson bivector.

Proof. It suffices to prove the statement on an affine open subset. Observe that $\operatorname{Ext}_{A}^{\circ}(B, B)$ is computed by the subcomplex

$$
C^{\bullet-1}\left(A, \operatorname{End}_{\mathbb{C}} B\right) \subset C_{A, B}^{\bullet}
$$

The differential $\delta^{\prime}$ of $\gamma_{B}: A^{\otimes(n-1)} \rightarrow$ End $_{\mathbb{C}} B$ is explicitly given by

$$
\delta^{\prime}\left(\gamma_{B}\right)=\left[\alpha_{A} \oplus \alpha_{B}, 0 \oplus \gamma_{B}\right]: A^{\otimes n} \rightarrow \operatorname{End}_{\mathbb{C}} B
$$

where [., $\cdot]$ is the Lie bracket on $C_{A, B}^{n}$ introduced in Section 2.2. Now a straightforward calculation finishes the proof.

5.3. Symplectic 2-form need not be locally exact. The following construction of a large class of examples of algebraic closed differential forms which are not locally exact in ètale topology was explained to us by A. Beilinson.

Let $X$ be a smooth algebraic variety, and $\omega$ a non-zero holomorphic $i$-form on $X$. We assume, in addition, that for some compactification of $X$ the form $\omega$ has logarithmic singularities at infinity (it is then automatically closed thanks to Deligne results [3]). In any case, one can merely assume that $X$ is itself a projective variety.

Claim 5.3.1. For any ètale morphism $\pi: U \rightarrow X$, the $i$-form $\omega_{U}=\pi^{*} \omega$, on $U$, is not exact.

Proof. Let $X \hookrightarrow Z$ be a compactification such that $Z \backslash X$ is a divisor with normal crossings. By Hironaka's resolution of singularities, one can find a similar compactification $U \hookrightarrow T$ such that the map $\pi: U \rightarrow X$ extends to a morphism $T \rightarrow Z$. It is known that in such a case, the $i$-form $\omega_{U}$ has logarithmic singularities at $T \backslash U$. Furthermore, this form is clearly non-zero since its restriction to $U$ is non-zero. The map sending a differential form $\beta$ on $T$ with logarithmic singularities at $T \backslash U$ to $[\beta] \in H^{i}(U)$, the corresponding de Rham cohomology class, is known to be injective, by $[\mathrm{De}]$. So, the class $\left[\omega_{U}\right] \in H^{i}(U)$ is non-zero. We conclude that $\omega_{U}$ can not be an exact form, as claimed.

The above result produces examples of smooth symplectic varieties (e.g. $X$ an abelian surface) such that the corresponding symplectic 2 -form cannot be made exact by passing to any ètale open subset. 
Acknowledgments. We are grateful to Vasiliy Dolgushev for his suggestion to use the shuffle product and to Sasha Beilinson for the construction reproduced in $\S 5.3$. We are indebted to Lev Rozansky for many patient explanations of his ongoing (partially unpublished) work with Anton Kapustin.

The work of the first author was supported in part by the Sloan Research Fellowship and the work of the second author was supported in part by the NSF grant DMS-0601050.

\section{References}

[1] V. Baranovsky, V. Ginzburg, and J. Pecharich, Deformation of line bundles on coisotropic subvarieties (2009). Preprint arXiv:0909.5520.

[2] K. Behrend and B. Fantechi, Gerstenhaber and Batalin-Vilkovisky structures on Lagrangian intersections, in Algebra, Arithmetic and Geometry: Manin Festschrift, Volume I., Vol. 269 of Progress in Mathematics, Birkhäuser (2009).

[3] P. Deligne, Théorie de Hodge. II, Inst. Hautes Études Sci. Publ. Math. (1971), no. 40, 5-57.

[4] Y. Frégier, M. Markl, and D. Yau, The $L_{\infty}$-deformation complex of diagrams of algebras, New York Journal of Math (2009), no. 15, 353-392.

[5] M. Gerstenhaber and S. D. Schack, On the deformation of algebra morphisms and diagrams, Trans. Amer. Math. Soc. 279 (1983), no. 1, 1-50.

[6] A. Grothendieck, Éléments de géométrie algébrique. IV. Étude locale des schémas et des morphismes de schémas IV, Inst. Hautes Études Sci. Publ. Math. (1967), no. 32, 361.

[7] A. Kapustin and L. Rozansky, Three-dimensional topological field theory and symplectic algebraic geometry II (2009). Preprint arXiv:0909.3643.

[8] M. Kontsevich, Deformation quantization of Poisson manifolds, Lett. Math. Phys. 66 (2003), no. $3,157-216$.

[9] J.-L. Koszul, Crochet de Schouten-Nijenhuis et cohomologie, Astérisque (1985), no. Numero Hors Serie, 257-271. The mathematical heritage of Élie Cartan (Lyon, 1984).

[10] J.-L. Loday, Cyclic homology, Vol. 301 of Grundlehren der Mathematischen Wissenschaften [Fundamental Principles of Mathematical Sciences], Springer-Verlag, Berlin, second edition (1998), ISBN 3-540-63074-0. Appendix E by María O. Ronco, Chapter 13 by the author in collaboration with Teimuraz Pirashvili.

[11] D. O. Orlov, Triangulated categories of singularities and D-branes in Landau-Ginzburg models, Tr. Mat. Inst. Steklova 246 (2004), no. Algebr. Geom. Metody, Svyazi i Prilozh., 240-262.

[12] C. A. Weibel, An introduction to homological algebra, Vol. 38 of Cambridge Studies in Advanced Mathematics, Cambridge University Press, Cambridge (1994), ISBN 0-521-43500-5.

Department of Mathematics, University of California at Irvine, Irvine, CA 92697, USA

E-mail address: vbaranov@math.uci.edu

Department of Mathematics, University of Chicago, Chicago, IL 60637, USA.

E-mail address: ginzburg@math.uchicago.edu 